XIV.

Aus dem Laboratorium der II. med. Klinik der Kgl. Charité, Berlin.

\title{
Die Aenderung der Blutalkalescenz beim Pankreasdiabetes unter dem Einfluss von Muskelkrämpfen.
}

Von

\author{
Max Sass, \\ Medicinalpraltikant.
}

Eine derjenigen Fragen, deren Studium noch vertieft werden muss, ist die Frage der Reaction des Blutes; ganz besonders gilt das von der Reaction des Diabetikerblutes. Wissen wir doch, dass z. B. der Exitus des Diabetikers unter dem Stigma der Säurevergiftung erfolgt, infolgedessen sollte man erwarten, dass, wenn auch das Blut ein starkes Neutralisationsbestreben hat, sich doch Vorgänge am Blute abspielen, die auf die Reaction des Blutes einen Einfluss haben.

Früher hat man das Blut für alkalisch angesehen und hat unter Alkalescenz des Blutes die Eigenschaft des Blutes verstanden, gegen Lackmus oder Lackmoid alkalisch zu reagieren. Statt dieser titrimetrischen Methode besteht heute die Möglichkeit, direkt den Alkalescenzgrad des Blutes durch die Ermittelung des Hydroxylionengehaltes zu bestimmen. Wenn man sich dieser Methode bedient, so stellt es sich nach R. Höber (1) heraus, dass der Hydroxylionengehalt des normalen Blutes ungefähr gleich dem der Wasserstoffionen ist, $d$. h., dass das Blut physico-chemisch eine so gut wie neutrale Flüssigkeit ist. Mlan nennt das die actuelle Reaction. Diese actuelle Reaction des Blutes kann durch einige Factoren beeinflusst werden. Hierher gehört der Gehalt des Blutes an Kohlensäure. Höber gibt an, dass das Blut zwar innerhalb der physiologischen Sehwankungen des $\mathrm{CO}_{2}$-Gehaltes annähernd neutrale Reaction beibehält, dass aber das venöse Blut doppelt so viel II+-Ionen enthalten kann, als das arterielle. Das würde mit anderen Worten besagen, dass das venöse Blut durch den Gehalt an $\mathrm{CO}_{2}$ saurer wäre, als das arterielle. Auch das I lebensalter spielt insofern eine Rolle bei der actuellen Reaction des Blutes, als der $\mathrm{H}+$-Ionengehalt des Blutes von Erwachsenen am geringsten, der von Säuglingen und besonders lebensschwachen, frühgeborenen Säuglingen am höchsten ist. Luftverdünnung scheint den $\mathrm{H}^{+}$-Gehalt des Blutes etwas $\%$ erhöhen.

Beim Coma diabeticum hat man nun vermittels der Titrationsmethode meist eine Abnahme der Blutalkalinität festgestellt. Nun aber hat Benedict (2) den Hydroxylionengehalt bestimmt und gefunden:

1. dass der Gehalt des Diabetikerblutes an $\mathrm{OH}$ sich auch in den Fällen mit abnormer Säureproduction nicht von der Norm unterscheidet, d. h. dem $\mathrm{OH}$-Gehalt nach das Blut eine neutrale Flüssigkeit ist;

2. dass bei dem Coma diabeticum ein Absinken des $\mathrm{OH}^{--}$-Gehaltes unter die Norm stattfinden kann, aber nicht constant vorkommt. Selbst 
in den Fällen mit tatsächlich niedrigem $\mathrm{OH}$-Gehalt bewegen sich die Werte hart an der Grenze der neutralen Reaction.

Das Blıt hat also die Tendenz, die doch zweifellos abnorme Säurebildung durch vermehrte Alkaliabgabe zu neutralisieren. Dies geht auch aus der Arbeit von $\Lambda$. Szilli (3) hervor, welcher Tieren intravenös Säuren injicierte und fand, dass die Alkalinität des Blutes mit der zugeführten Säuremenge nicht proportional sinkt. Dadurch, dass dieselbe Säuremenge anfangs die Alkalinität des Blutes erheblich mehr herabsetzt wie später, schliesst er, dass das Alkali, welches der Körper zur Neutralisierung der eingeführten Säure braucht, nicht allein aus dem Blute, sondern auch aus den Zellen des übrigen Organismus stammt.

Die Bestimmung des Hydroxylionengehaltes ergibt also ein Resultat, wolches dem mit IIlle der Titrationsmethode und der $\mathrm{CO}_{2}$-Bestimmung gerade entgegengesetzt ist und, wenn sie allein ausschlaggebend wäre, die ganze Acidosetheorie hinfällig machen würde.

Nun ist aber das Alkali des Blutes in diesem in zwei Formen vorhanden: einmal als freies, chemisch locker gebundenes, hauptsächlich als kohlen- und phosphorsaures Alkali, und zweitens als Alkali, welches mit Eiweissstoffen des Blutes feste, unlösliche Verbindungen eingegangen ist. Bei der Bestimmung der Hydroxylionen können natürlich nur die freien $\mathrm{H}+$-Ionen bestimmt werden, und da diese, wie Joew y-7untz (4) gezeigt haben, gegenüber den festgebundenen erheblich in der Minderzahl sind, so erhalten wir durch die Bestimmung des Hydroxylionengehaltes keine exacte Aufklärung über die Reaction des Diabetikerblutes.

Um die Alkalescenzänderung des Blutes zu ermitteln, bleibt uns also nur eine indirecte Methode, und diese besteht entweder darin, dass man bestimmt, um wieviel der $\mathrm{CO}_{2}$-Gehalt des Blutes abgenommen hat (Verdrängung der $\mathrm{CO}_{2}$ durch stärkere Säuren) oder darin, dass man das Blut gegen Lackmus oder Lacknioid austitriert; es muss aber dann unter letzteren Verhältnissen sowohl das locker- wie festgebundene Alkali bestimmt werden.

Wir haben uns nun, auf Veranlassung von Professor Brugsch, die Frage vorgelegt: ist eine Aenderung der 4 lkalescenz des J3lutes unter solchen Bedingungen zu constatieren, unter denen erfahrungsgemüss organische Säuren, z. B. Hilchsäure, im Blute aufzufinden sind, und weiter haben wir uns die Frage vorgelegt, ob ein Hund, bei dem eine Aenderung des Kohlehydratstoffwechsels durch Exstirpation des Pankreas bewirkt ist, unter ähnlichen Verhältnissen Abweichungen hinsichtlich dieser Alkalinitätsänderung zeigt.

7u diesem / $/$ wecke exstirpierten wir einer Anzahl von Hunden das Pankreas total und setzten sie, ebenso wie eine Anzahl normaler Hunde, unter Strychninwirkung. Das Blut wurde alsdann vor und während der Krämple und nach dem Tode untersucht, mittelst der Alkalescenzbestimmung von L Loewy und Zuntz, bei der das Verhältnis zwischen fest und locker gebundenom Alkali ausgewertet wird.

Wir beschreiben zunächst die Versuche und werden daran die daraus gezogenen Schlussfolgerungen anschliessen. 


\section{Metlıodik der Versuche.}

Die Yethode, welche ich bei meinen Verswen anwendete, ist dieselbe, wie sie K. Brandenburg in seiner Arbeit ,Ueber das diffusible Alkali und die Alkalispannung des Blutes in Krankheiten " angibt. Sie ist eine Modification der zuersi von Loewy und Zuntz mitgeteilten Methode und gestaltet sich folgendermassen: Dem Hunde werden etwa $80-100 \mathrm{ccm}$ Blut abgelassen, und dieses wird durch Schlagen defibriniert. Hiervon werden 5 cem mit der 4 fachen Henge Aqua dest. versetzt und dadurch lackfarben gemacht. Dann wird die Alkalescenz durch Titration mit $1 / 25$ Normalweinsäure gegen Lackmoidpapier bestimmt. Hierauf werden $20 \mathrm{ccm}$ Blut in einen feuchten Pergamentschlauch von etwa $15 \mathrm{~cm}$ Höhe und $4 \mathrm{~cm}$ Lichtung gebracht. Der Pergamentschlauch ist an einem Ende durch einen Gummistopfen verschlossen und muss vor jeder Benutzung auf seine Dichtigkeit hin geprüft werden. Sodann wird er in einen Glascylinder von etwa $20 \mathrm{~cm}$ Höhe und $5 \mathrm{~cm}$ Lichtung hineingestellt, in dem sich $50 \mathrm{ccm}$ einer mit isotonischer Kochsalzlösung hergestellten Natronlauge befinden, deren Procentgehalt an Alkali den fünften, bzw. den sechsten Teil von dem des Blutes beträgt. Das Blut im Pergamentschlauch muss öfters mit einem dicken Glasstabe umgerührt werden. Nach 24 stündigem Stehen in einem nicht zu warmen Raume wird das Alkali der Innen- und Aussenflüssigkeit wie vorhin titriert.

Sämtliche Titrationen wurden doppelt ausgeführt. $\Lambda$ ls Indicator benutzte ich das nach Loewyscher Vorschrift von Apotheker Dr. Wartenberg hergestellte Iackmoidpapier.

Versuch I. Einem ca. $6-7 \mathrm{~kg}$ schweren Hunde wurden ca. $100 \mathrm{ccm}$ Blut abgelassen zu Versuch I a.

Nach einigen Tagen wurde $i \mathrm{hm} 1 \mathrm{mg}$ Strychnin subcutan injiciert. 12 Minuten später trat der erste Krampf auf. Noch während des Krampfes wurde angefangen, Blut zu entnehmen zu Versuch Ib $c$. Die Blutentnahme dauerte 13 Minuten. Während dieser Zeit traten 3 Krämpfe auf. Hs wurde dann wiederum $1 \mathrm{mg}$ Strychnin injiciert, darauf folgten nach 7 Minuten Krämpfe und nach weiteren 2 Minuten der Tod. Nach dem Tode wurde sofort der Bauch eröffnet und Blut aus der Vena cava inf. zu VersuchIbp entnommen.

Die Zeit von der 1. Injection bis zum Tode betrug 43 Minuten; dem Hunde wurden im ganzen $2 \mathrm{mg}$ Strychninum nitricum injiciert.

\section{Versueh I: Am Hunde ohne Pankreas-Exstirpation.}

a) Vorder Strychnin-Injection.

Blutalkalescenz für $100 \mathrm{ccm}$ : $416,0 \mathrm{mg} \mathrm{NaOH}$. verfärbt.

Dialyse: Dauer 24 Stunden; kein Fäulnisgeruch, Aussenflüssigkeit z. T. gelblich

\begin{tabular}{|c|c|c|c|c|c|c|c|c|c|c|}
\hline & \multicolumn{4}{|c|}{ ccm Flüssigkeit } & \multicolumn{4}{|c|}{$\begin{array}{c}\text { ccm n/25 Weinsäure zur } \\
\text { Neutralisation von } 10 \mathrm{ccm} \\
\text { Flüssigkeit }\end{array}$} & \multirow{2}{*}{\multicolumn{2}{|c|}{$\begin{array}{l}\text { Alkaleseenz der } \\
\text { Aussenflussigkeit } \\
\text { zur Alkalescenz } \\
\text { des Blutes ( } \mathbf{A}: \mathbf{J})\end{array}$}} \\
\hline & \multicolumn{2}{|c|}{ aussen } & \multicolumn{2}{|c|}{ innéen } & \multicolumn{2}{|c|}{ aussen } & \multicolumn{2}{|c|}{ innen } & & \\
\hline & vor & nach & vor & nach & vor & nach & vor & vach & vor & nach \\
\hline I. & 50,0 & 48,0 & 20,0 & 22,0 & 5,0 & 4,0 & 25,0 & 24,5 & $1: 5$ & $1: 6,1$ \\
\hline lI. & 50,0 & 46,0 & 20,0 & 21,5 & 4,2 & 3,6 & 25,0 & 23,4 & $1: 6$ & $1:(j, 5)$ \\
\hline
\end{tabular}


b) Nach dor Strychnin-Injection.

(c) Während der Krämpfe.

Blutalkalescenz für $100 \mathrm{ccm}: 256,0 \mathrm{mg} \mathrm{NaOH}$.

Dialyse: Dauer 24 Stunden; kein Fäulnisgeruch; Aussonflüssigkeit klar.

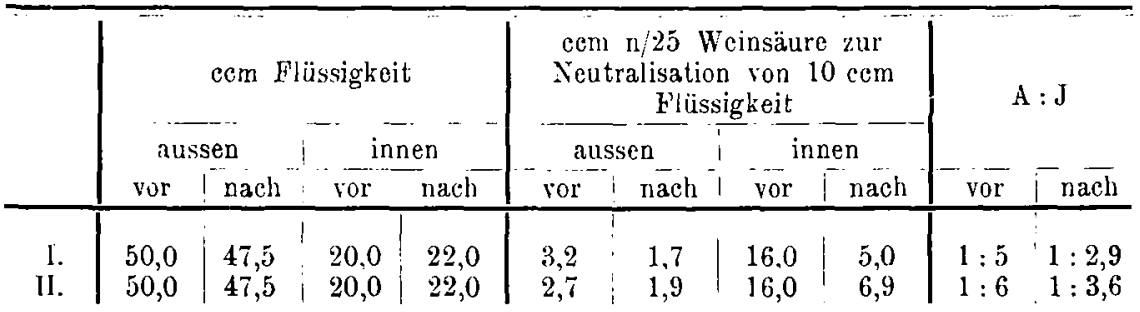

ß) Nach dem T'ode.

Blutalkalescenz für $100 \mathrm{ccm}$ : $208,0 \mathrm{mg} \mathrm{NaOH}$.

Dialyse: Dauer 24 Stunden; kein Fäulnisgeruch, Aussenflüssigkeit klar.

\begin{tabular}{|c|c|c|c|c|c|c|c|c|c|c|}
\hline & \multicolumn{4}{|c|}{ ccm Flüssigkeit } & \multicolumn{4}{|c|}{$\begin{array}{l}\text { cem nos Weinsiure zur } \\
\text { Neutralisation von } 10 \mathrm{ccm} \\
\text { Flüssiglieit }\end{array}$} & \multicolumn{2}{|c|}{$A: J$} \\
\hline & $\mathrm{au}$ & sen & - in & & & & & nen - & & \\
\hline & $\overline{\text { vor }}$ & nach & vor & nash & vor & nach & vor & nach & yor & nach \\
\hline I. & 50,0 & 46,5 & 20.0 & 23,0 & 2,6 & 1,8 & 13,0 & 8,2 & $1: 5$ & $1: 4, \bar{j}$ \\
\hline Il. & 50,0 & 47,0 & 20,0 & 22,5 & 2,2 & 1,5 & 13,0 & 7,3 & $1: 6$ & $1: 4,8$ \\
\hline
\end{tabular}

Versuch II. Einem ca. $6 \mathrm{~kg}$ schweren Hunde wurden ca. $80 \mathrm{ccm}$ Blat entnommen zu Versuoh II a. Darauf wurde ihm $1 \mathrm{mg}$ Strychnin subcutan injiciert. Nach 8 Minuton trat der erste Krampf auf. Es wurde sofort Blut entnommen zu Versuch IIb ce. Die Blutentnahme dauerte 15 Minuten. Während dieser Zeit traten mehrere kleinere Krämpfe auf. 30 Minuten nach der ersten Injection wurde eine zweite gemacht von $1 / 2 \mathrm{mg}$, eine dritte ebensolche nach weiteren 20 Minuten. Während dieser Zeit wurden die Krämpfe heftiger und die Blutentnahme wurde fortgesotzt. 20 Minuten nach der dritten Injection wurde eine vierte gemacht, wiederum $1 / 2 \mathrm{mg}$. Nach 7 Minuten trat unter heftigen Krämpfen der 'lod ein. Nach dem 'Tode wurde sofort der Bauch eröfinet und aus der Vena cava inf. Blut zu Vorsuch IIb $\beta$ entnommen.

Die Zeit von der 1 . Injection bis zum Tode betrug 77 Minuten; es wurden insgesamt $21 / 2 \mathrm{mg}$ Strychninum nitricum injiciert.

Versuch II: Am Hunde ohne Pankreas-Exstirpation.

a) Vor der Strychnin-Jnjection.

Blutalkalescenz für $100 \mathrm{ccm}: 374,4 \mathrm{mg} \mathrm{NaOH}$.

Dialyse: Dauer 24 Stunden: Aussenflüssigkeit klar, kein Fäulnisgeruch.

\begin{tabular}{|c|c|c|c|c|c|c|c|c|c|c|}
\hline & & $\mathrm{cm} \mathrm{H}$ & sigk & & $\begin{array}{l}\mathrm{cc} \\
\mathrm{Ne}\end{array}$ & $\begin{array}{r}/ 25 \\
\text { lisati } \\
\text { Flü }\end{array}$ & $\begin{array}{l}\text { nsäl } \\
\text { von } \\
\text { keit }\end{array}$ & $\begin{array}{l}\text { zur } \\
\mathrm{cem}\end{array}$ & & \\
\hline & & & $\ldots$ & & $\ldots$ & In & & & & \\
\hline & vor & nach & vor & nach & vor & nach & vor & nach & vor & nach \\
\hline I. & 50,0 & 46,0 & 20,0 & 23,5 & 4,7 & 3,6 & 23,4 & 24,1 & $1: 5$ & $1: 6,7$ \\
\hline Il. & 50,0 & 46,0 & 20,0 & 24,0 & 3,9 & 3,$1 ;$ & 23,4 & 23,9 & $1: 6$ & $1: 6,6$ \\
\hline
\end{tabular}


b) Nach der Strychain-Injection.

a) Während der Krämpfe.

Blutalkalescenz für $100 \mathrm{ccm}: 300,8 \mathrm{mg} \mathrm{NaOH}$.

Dialyse: Dauer 24 Stunden; Aussenflüssigkeit klar, kein Fäulnisgeruch.

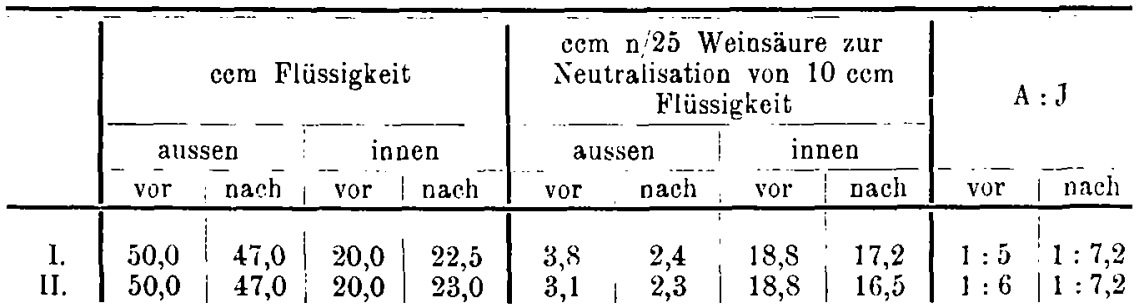

ß) Nach dem Tode.

Blutalkalescenz für $100 \mathrm{ccm}$ : $185,6 \mathrm{mg} \mathrm{NaOH}$.

Dialyse: Dauer 24 Stunden; Aussenflüssigkeit klar, kein Fäulnisgeruch.

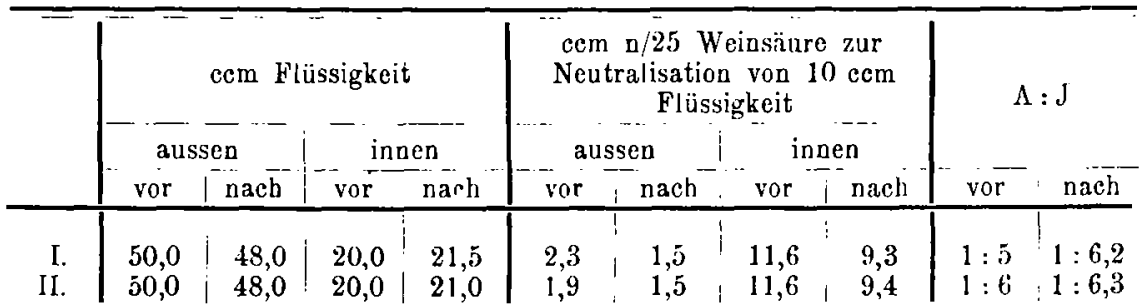

Versuch III. Einem $7 \mathrm{~kg}$ schweren Hunde wurde das Pankreas total exstirpiert. Bis zum folgenden Tage hatte der Hund keinen Urin gelassen. Frst bei der nun folgenden Strychninvergiftung gelang es, etwas Urin aufzufangen; bei der geringen Menge aber war es nicht möglich, einen Zuckergehalt sicher nachzuweisen. Vor der Strychnin-Injection wurde dem Hunde Blut abgelassen zu Versuch IIIa. Dann wurde ihm $1 \mathrm{mg}$ Strychnin subcutan injiciert, nach 26 Minuten wiederum $1 \mathrm{mg}$, nach weiteren 39 Minuten $1 / 2 \mathrm{mg}$, nach weiteren 15 Minuten und weiteren 24 Minuten wiederum jo $1 / 2$ mg. 7 Minuten nach der vorletzten Injection trat der erste Krampf auf. Daraufhin versuchte ich, dem Hunde Blut abzulassen. Es bestand jedoch ein derartiger Spasmus der Gefässe, dass eine Blutentnahme unmöglich war. 11 Minuten nach der letzten Injection wurde dem Hunde nocbmals $1 \mathrm{mg}$ Strychnin injiciert und nach 19 Minuten trat unter heftigen Krämpfen der Tod ein. Nach dem l'ode wurde sofort Blut aus der Vena cava inf. zu Versuch III b $\beta$ ontnommen.

Die Zeit von der 1. Injection bis zum Tode betrug 2 Stunden 12 Minuton; es wurden insgesamt $41 / 2 \mathrm{mg}$ Strychninum nitricum injiciert. Versuch IIl: Nach Pankreas-Exstirpation. Znckergehalt des Harns nicht
nachweisbar.

a) Vor der Strychnin-Injection.

Blutalkalescenz für $100 \mathrm{ccm}$ : $393,6 \mathrm{mg} \mathrm{NaOH}$.

Dialyse: Dauer 24 Stunden; Aussenflüssigkeit klar, kein Fäulnisgeruch.

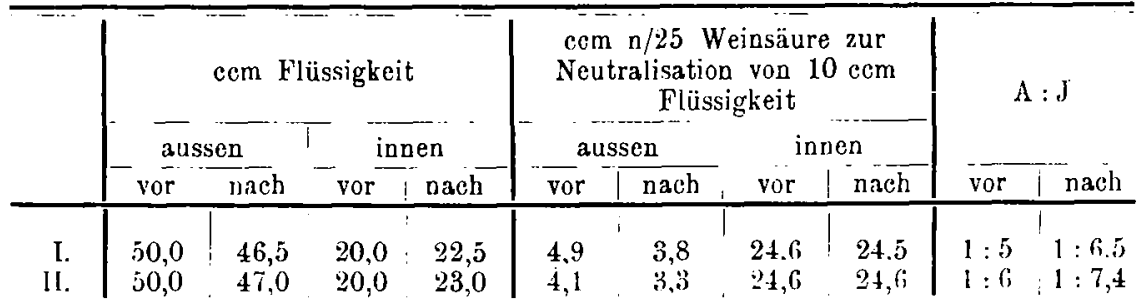


b) Nach der Strychnin-Injection.

a) Wälırend der Krämpfe war eine Blutentnahme wegen Spasmus der Gefässe nicht möglich.

ק) Nach dem Tode.

Blutalkalescenz für $100 \mathrm{ccm}$ : $316,8 \mathrm{mg} \mathrm{NaOH}$.

Dialyse: Dauer 24 Stunden; Aussenflüssigkeit klar, kein Fäulnisgeruch.

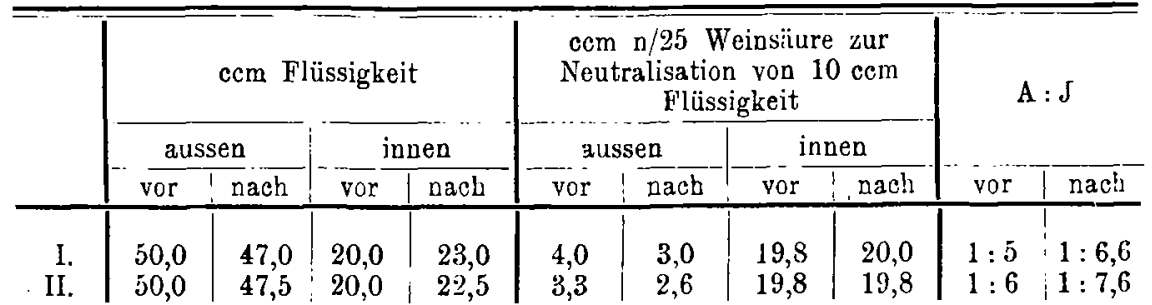

Versuch IV. Finom $91 / 2 \mathrm{~kg}$ schweren Hunde wurde das Pankreas exstirpiert. Als nach 2 Tagen der 'Zuckergehalt des Urins $3^{1 / 2} \mathrm{pCt}$. betrug, wurde dem Hunde Blut abgelassen zu Versuch IV a. Die Blutentnabme dauerte 15 Minuten. Darauf wurden dem Hunde $2 \mathrm{mg}$ Strychnin subcutan injiciert. Nach 15 und nach 30 Minuten erhielt er wiederum je $1 \mathrm{mg}$ Strychnin, also im ganzen $4 \mathrm{mg}$ in $1 / 2$ Stunde. 5 Minuten nach der letzten Injection trat der erste heftige Krampf auf, dem bald mebrere folgten. Während der Krämpfe wurde Blut entnommen zu Versuch IV bo. Diese Blutentaahme dauerte 31 Minuten. Sie wurde orschwert durch Spasmus der Gefässe. Nach weiteren ca. 15 Minuten erfolgte der Tod unter starken Krämpfen. Sofort nach dem Tode wurde aus der Vena cava inf. Blut zu Versuch IV $b \beta$ entnommen.

Die Zeit von der 1 . Injection bis zum Tode betrug 1 Stunde 22 Minuten, es wurden im ganzen $4 \mathrm{mg}$ Strychninum nitricum injicirt.

Versach IV: Nach Pankreas-Exstirpation. Zackergehalt des Harns 31/2 p(tt.

a) Vor der Strychnin-Injection.

Blutalkalescenz für $100 \mathrm{ccm}$ : $406,4 \mathrm{mg} \mathrm{NaOH}$.

Dialyse: Dauer 24 Stunden; Aussenflüssigkeit z. T. schwach gelblich vorfärbt, kein Fäulnisgeruch.

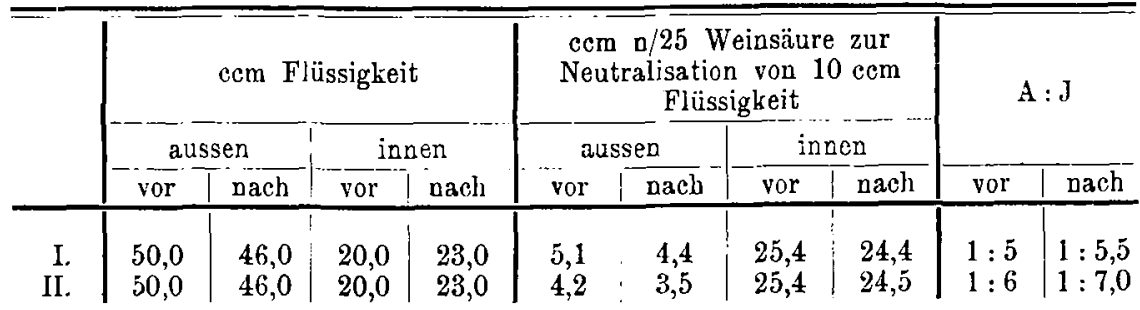

b) Nach der Strychnin-Injection.

c) Während dor Krämpfe.

Blutalkalescenz für $100 \mathrm{com}: 326,4 \mathrm{mg} \mathrm{NaOH}$.

Dialyse: Dauer 24 Stunden; Aussenflüssigkeit klar, kein Fäulnisgeruch.

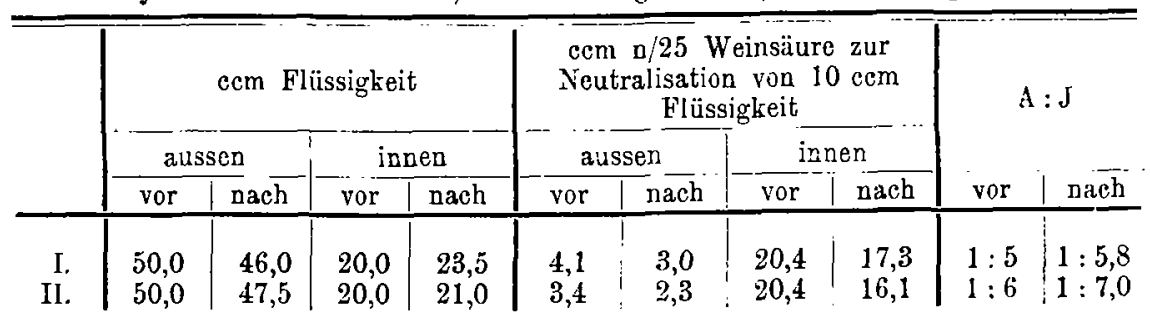

Zeitschrift f. exp. Tathologio u. Therapie. 15. Bd. 
$\beta)$ Nach dem Tode.

Blutalkalescenz für $100 \mathrm{ccm}: 265,6 \mathrm{mg} \mathrm{NaOH}$.

Dialyse: Dauer 24 Stunden; Aussenflüssigkeit klar, kөin Fäulnisgeruch.

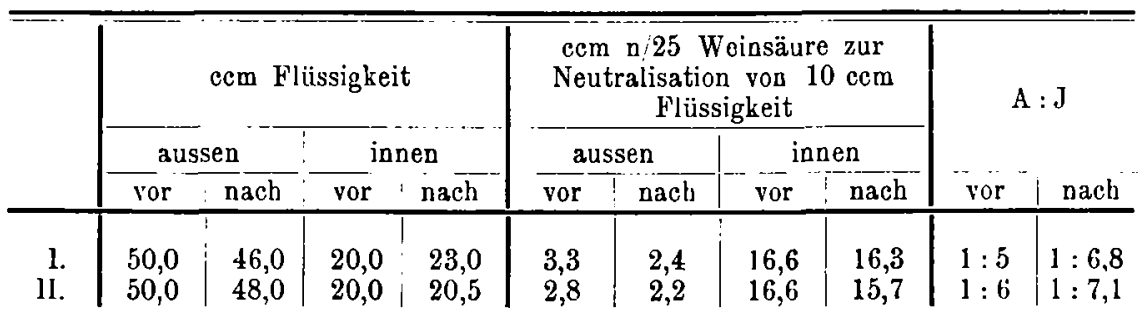

Versuch V. Einem 9-10 kg schweren Hunde wurde das Pankreas exstirpiert. Am näcbsten Tage enthielt der Harn 3 pCt. Daraufhin wurde dem IIunde Blut abgelassen zu Versuch Va. Die Blutentnahme dauerte ca. 45 Minuten. Hierauf wurden ihm $2 \mathrm{mg}$ Strychnin subcutan injiciert, nach 30 Minuten und nach weiteren 15 Minuten wiederum je $1 \mathrm{mg}$ und nach weiteren 20 Minuten noch $1 / 2 \mathrm{mg}$ Strychnin. 1 Minute nach der letzten Injection trat der erste grosse Krampf aut, dem in kurzen Abständen mehrere folgten. Nach dem ersten Krampfe wurde dem Hunde Blut entnommen zu Versuch Vb $\alpha$. Wegen des starken Spasmus der Gefässe war es nicht möglich, die gewöhnliche Blutmengo zu erhalten. Die Blutentnahme dauerte 28 Minuten, nach welcher Zeit der Tod unter heftigen Krämpfen eintrat. Nach dem Tode wurde aus der Vena cava inf. Blut zu Versuch Vb $\beta$ entnommen.

Die Zeit von der 1 . Injection bis zum Tode betrug 1 Stunde 34 Minuten; es wurden im ganzen $4^{1} / 2 \mathrm{mg}$ Strychnin injiciert.

Versnch V: Xach Pankreas-Exstirpation. Zuckergehalt les Harns 3 pet.

a) Vorder Strychnin-Injection.

Blutalkalescenz für $100 \mathrm{ccm}$ : $409,6 \mathrm{mg} \mathrm{NaOH}$.

Dialyse: Dauer 24 Stunden; Aussenflüssigkeit klar, kein Fäulnisgeruch.

\begin{tabular}{|c|c|c|c|c|c|c|c|c|c|c|}
\hline & \multicolumn{4}{|c|}{ cem Flüssigkeit } & \multicolumn{4}{|c|}{$\begin{array}{c}\text { ccm o/25 Weinsäure zur } \\
\text { Neutralisation von } 10 \mathrm{ccm} \\
\text { Flïssigkeit }\end{array}$} & \multirow{2}{*}{\multicolumn{2}{|c|}{$A: J$}} \\
\hline & \multicolumn{2}{|c|}{ aussen } & \multicolumn{2}{|c|}{ innen } & \multicolumn{2}{|c|}{ aussen } & \multicolumn{2}{|c|}{ innen } & & \\
\hline & vor & nach & vor & nach & vor & nach & vor & nach & vor & nach \\
\hline $\begin{array}{l}\text { I. } \\
\text { II. }\end{array}$ & $\begin{array}{l}50,0 \\
50,0\end{array}$ & $\begin{array}{l}45,5 \\
45,0\end{array}$ & $\begin{array}{l}20,0 \\
20,0\end{array}$ & $\begin{array}{l}24,0 \\
24,0\end{array}$ & $\begin{array}{l}5,1 \\
4,3\end{array}$ & $\begin{array}{l}4,1 \\
3,6\end{array}$ & $\begin{array}{l}25,6 \\
25,6\end{array}$ & $\begin{array}{l}25,0 \\
24,7\end{array}$ & $\begin{array}{l}1: 5 \\
1: 6\end{array}$ & $\begin{array}{l}1: 6,1 \\
1: 6,8\end{array}$ \\
\hline
\end{tabular}

b) Nach der Strychnin-Injection.

$\alpha)$ Während der Krämpfe.

Blutalkalescenz für $100 \mathrm{ccm}: 342,4 \mathrm{mg} \mathrm{NaOH}$.

Dialyse: Dauer 24 Stunden; Aussenflüssigkeit klar, kein Fäulnisgerucb.

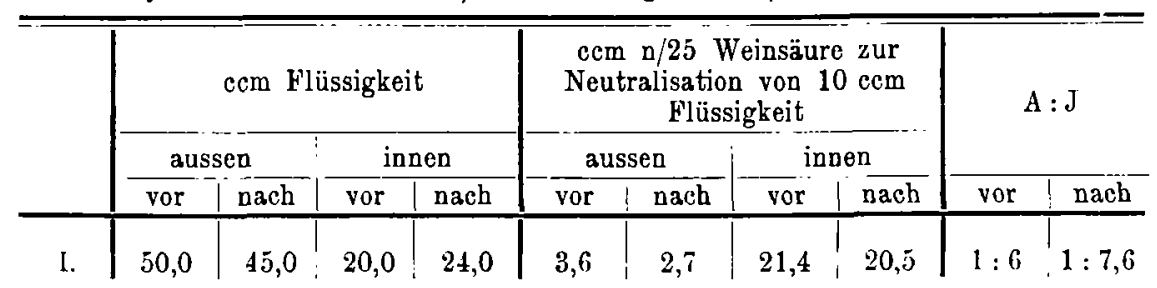


B) Nach dem Todo.

Blutalisalescenz für $100 \mathrm{ccm}: 281,6 \mathrm{mg}$ KaOHi.

Dialyse: Dauer 24 Stunden; Aussenflüssigkoit klar, kein Fäulnisgeruch.

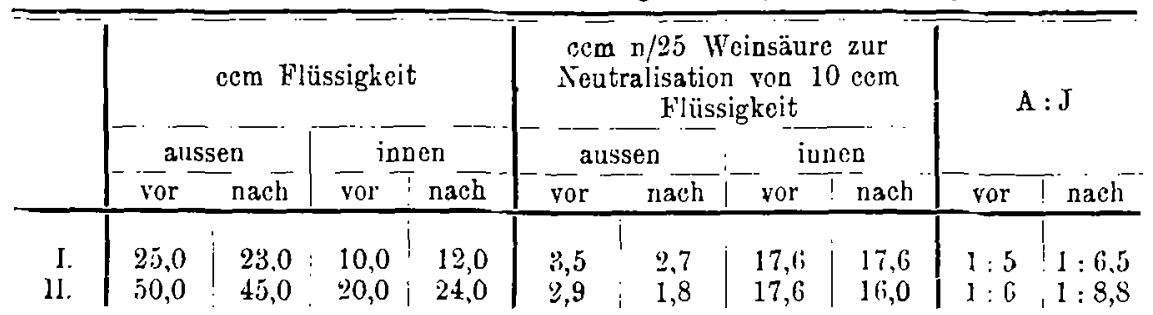

Wenn wir die Resultate nunmelsr hier zusammenfassen, so ergibt sich foigendes:

An oinem normalen Hunde, der nicht operiert worden war, zeigt sich an dem nüchtern untersuchten Blut, dass die Alkalescenz nach titrimetrischer Bestimmung sinkt, sobald durch Strychnin-lnjection Krämpfe hervorgerufen werden. Besonders tritt auch ein Sinken der Alkalescenz nach dem Tode ein. Die Ursache dieses Sinkens der Alkalescenz muss auf eine Production von Säure zurückzuführen sein (wahrscheinlich Milchsäure). Wurde den Hunden das Pankreas exstirpiert und die gleichsinnigen Versuche angestellt, so ergab sich allerdings auch ein Sinken der Alkalinität. Dieses Sinken betrug vielleicht $1 / 4$ des Wertes der Gesamt-Alkalinität, war aber quantitativ weit geringer als das Sinken der A!kalinität boim nichtoperierten Hunde. Fin gleiches Resultat ergab der Versuch 4 und 5. Auch hier war ein Sinken der Alkalinität zu constatieren, doch nicht so erheblich wie in den Normalversuchen. Wenn wir diese Versuche beurteilen, dann müssen wir doch sagen, dass in der Tat durch Entstehung von Säuren bei der Arbeit eine Herabminderung der Alkalinität des Blutes möglich ist, dass aber die Pankreasexstirpation eine derartige Herabminderung nicht bewerkstelligt. Das kann auf der Unmöglichkeit beruhen, hier den Zucker in vollem Masse zu zerstören, wie es der gesunde Hund tut; wissen wir doch, dass zur Muskelarbeit zum mindesten (wenn nicht etwa allein) Kohlehydrate in Umsatz gebracht werden, und dass da, wo Krämpfe auftreten, reichlich Nilchsäure produciert wird. - Es ist nun für die geringere Herabsetzung der Alkalescenz nicht etwa die Exsticpation des Pankreas verantwortlich zu machen in dem Sinne, dass diese die Strychninkrämpfe nicht zustande kommen lässt; in Gegenteil haben die Hunde weit grössere Krämpfe aufgewiesen als die nicht operierten. Man hätte danach also gerade ein starkes Herabsinken der Alkalinität erwarten können.

Wir kommen daher zu dem Schluss, dass in der Tat ein Säurcnzuwachs, wie er durch Krämple beim gesunden Tier entsteht, eine Herabsetzung der Alkalinität des Blutes hervorruft, ohne dass der Hydroxylionengehalt des Blutes sich wesentlich zu ändern braucht. Dass er sich wirklich nicht erheblich ändert, beweisen Versuche Iwanorfs, die in g]ejcher Richtung angestellt sind und in der vorangehenden Arbeji niedergelegt sima. 
378 Max Sass, Die Aenderung der Blutalkalescenz beim Pankreasdiabetes usw.

Weiter zeigen unsere Versuche, dass die Pankreasexstirpation zwar nicht die Herabsetzung der Alkalinität des Blutes bei Auftreten von Krämpfen hindert, dass aber trotz vermehrter Krämpfe die Herabsetzung der Alkalinität eine geringere ist als gegenüber der Norm, und wir deuten diese geminderte Herabsetzung am ehesten als die Folge einer gewissen Insufficienz der Kohlehydratzerlegung, bedingt durch die Pankreasexstirpation.

Die Arbeit ist auf Veranlassung und unter Leitung von Prof. Dr. Brugsch ausgeführt.

\section{Literatnr.}

1) R. Höber, Physikal. Chemie des Blutes und der Lymphe. Handbuch der Biochemie des Menschen und der Tiere. 1909. 2. Bd. 2. Teil. S. 10.

2) H. Benedict, Der Hydroxylionengehalt des Diabetikerblutes. Arbeiten auf dern Gebiete d. chem. Physiologie, herausgegeb. von Dr. F. Tangl. 1906. 3. H. S. 106.

3) A.Szilli, Experimentelle Untersuchungen über Säureintoxikationen. Ebenda. S. 82.

4) I oew y-Zuntz, Ueber die Bindung der Alkalien in Serum und Blutkörperchen. Pflügers Archiv. 1894. 58. Bd. 